\title{
Los estudiantes politécnicos en México y sus percepciones sobre los miedos que los obstaculizan para emprender
}

\author{
Polytechnic students in Mexico and their perceptions \\ about the fears that hinder them to undertake entrepreneurship \\ Claudia Alejandra Hernández Herrera \\ María del Pilar Monserrat Pérez Hernández
}

\begin{abstract}
RESUMEN
El emprendimiento permite activar las economías de los países, genera empleos, desarrolla nueva tecnología e incrementa el nivel de vida y bienestar de los ciudadanos. El objetivo de este artículo fue analizar las percepciones que tienen los jóvenes politécnicos con relación al fenómeno del emprendimiento y sus posibles obstáculos. Es un estudio transversal; la obtención de datos se realizó por medio de un cuestionario, y participaron 9,626 estudiantes de los tres niveles educativos del Instituto Politécnico Nacional. Se realizó un estudio descriptivo y se emplearon modelos lineales generalizados que permiten el análisis multivariable. Se encontró que apenas el 15\% de los estudiantes ha participado en actividades de emprendimiento, además de que solo el $25 \%$ de los jóvenes de enseñanza media y superior ha tenido negocios (pero únicamente el 11\% de estos siguen operando). El miedo a fracasar se detectó con mayor prevalencia en las mujeres que estudian el bachillerato o licenciatura, aunado a que la sensación de fracaso se presentó en jóvenes que provienen de familias de sectores vulnerables. Se concluye que el instituto tiene que poner mayor atención a las mujeres para capacitarlas, así como mantener y reforzar sus acciones orientadas a la educación empresarial y formación del espíritu empresarial.
\end{abstract}

Palabras claves: emprendimiento, educación superior, obstáculos para emprender, mentalidad emprendedora, espíritu empresarial.

\section{ABSTRACT}

Entrepreneurship allows economy growth of countries, creates jobs, develops new technology and increases the standard of living and well-being of citizens. The objective of this article was to analyze the perceptions that young polytechnics students have in relation to the phenomenon of entrepreneurship and its possible obstacles. It is a cross-sectional study; data was obtained through a questionnaire, and 9,626 students from the three educational levels at Instituto Politécnico Nacional participated. A descriptive study was carried out and generalized linear models that allow multivariate analysis were used. It was found that only $15 \%$ of the students have participated in entrepreneurial activities, in addition to that only $25 \%$ of young people from secondary and higher education have had businesses (but only $11 \%$ of these continue to operate). The fear of failure was detected with greater prevalence in women who study high school or undergraduate, coupled with the fact that the feeling of failure was presented in young people who come from families in vulnerable sectors. It is concluded that the institute must pay greater attention to women to train them, as well as to maintain and reinforce its actions aimed at business education and entrepreneurship training.

Keywords: entrepreneurship, higher education, obstacles to entrepreneurship, entrepreneurial mindset, entrepreneurial spirit. 


\section{INTRODUCCIÓN}

De acuerdo con el reporte global del Monitor de Emprendimiento 2019, las percepciones y actitudes y la actividad temprana hacia el emprendimiento indican que el 68\% de hombres y de mujeres en México señaló estar de acuerdo en que el emprendimiento es una buena opción deseable de carrera, además el 48\% de hombres y el 44\% de mujeres en el país conoce a alguien que ha emprendido un negocio en los últimos dos años. Otro hallazgo: el 73\% de los hombres y el 68\% de las mujeres perciben que tienen los conocimientos, las habilidades y la experiencia necesaria para iniciar un negocio. Por otra parte, el 47\% de las mujeres y el 48\% de los hombres tienen miedo al fracaso que puede generarse en el proceso del emprendimiento (Alonso, Barrera, Barrón, Berrelleza, Carbajal, Cortés, Jiménez, Pouzou y Rehák, 2019).

Cada vez es más difícil ignorar que los ambientes de incertidumbre económica que enfrentan muchas naciones requieren mejorar la situación de empleo, uno de esos caminos es impulsar el emprendimiento desde las instituciones de educación superior (Vaquero-García, Ferreiro-Seoane y Álvarez-García, 2017). Sin embargo, de acuerdo con Ekore y Okekeocha (2012), el espíritu empresarial es una alternativa viable y atractiva para el desempleo juvenil, pero los egresados universitarios son reacios y no ven el emprendimiento como una opción viable de carrera. La proporción de estudiantes graduados que construyen sus propias empresas es insignificante, por lo que se debe poner atención a la educación de talentos en las instituciones de educación superior (Yao, Xu y Xu, 2013).

La literatura científica ha señalado que los obstáculos para el desarrollo y promoción del espíritu empresarial son multidimensionales: en principio se tiene el desajuste entre los planes educativos y las necesidades del mercado de trabajo; además del empleo de métodos de enseñanza inconsistentes, escaso énfasis en el aprendizaje de habilidades científicas, falta de contenidos educativos actualizados, excesivos cursos teóricos, uso de métodos tradicionales, falta de contratación de instructores con

Claudia Alejandra Hernández Herrera. Profesora-investigadora del Instituto Politécnico Nacional, México. Se encuentra adscrita a la Unidad Profesional Interdisciplinaria de Ingeniería y Ciencias Sociales y Administrativas (UPIICSA), sección de Estudios de Posgrado e Investigación. Pertenece al Sistema Nacional de Investigadores, Nivel 1. Es coordinadora del Programa de Maestría en Administración, que pertenece al Programa Nacional de Posgrado de Calidad (PNPC) del ConACyT y profesora del Núcleo Académico de la maestría en Ciencias en Estudios Interdisciplinarios para la Pequeña y Mediana Empresa. Correo electrónico: cahernandezh@ipn.mx. ID: https://orcid.org/0000-0002-4060-2941.

María del Pilar Monserrat Pérez Hernández. Centro de Investigaciones Económicas, Administrativas y Sociales del Instituto Politécnico Nacional, México. Es doctora en Economía y Gestión de la Innovación y Política Tecnológica. Miembro del Sistema Nacional de Investigadores, Nivel 1, y del RCEA del ConAcyт. Fue directora del Centro de Incubación de Empresas de Base Tecnológica del IPN (septiembre 2015 a febrero 2018), encargada de la Oficina de Transferencia de Tecnología del IPN (junio 2010 a febrero 2013). Actualmente es coordinadora de la maestría en Política y Gestión del Cambio Tecnológico del CieCAs y coordinadora de la Red de Desarrollo Económico, ambos del IPN. Correo electrónico: mpperez@ipn.mx. ID: https://orcid. org/0000-0003-1352-6004. 
experiencia, mínima interacción entre la universidad y la empresa, relaciones poco adecuadas entre el sistema de educación superior y los sectores económicos líderes de la sociedad, los profesores no han adquirido habilidades específicas para la educación empresarial, los contenidos solo se ofrecen de manera teórica y no práctica, así como bajo número de emprendedores exitosos (Karimi, Chizari, Biemans y Mulder, 2010; Fallah, Razavi, Rezvanfar y Kalantari, 2014; Kozlinska, 2011; Lima, Almeida, Maria, Nassif y da Silva, 2012; Nwekeaku, 2013; Xianming, 2013; Martínez y Ventura, 2020).

De acuerdo con Pérez (2020), el IPN en el 2004 creó el Centro de Incubación de Empresas de Base Tecnológica (CIEBT), cuyo modelo de incubación robusto ha sido replicado por 500 incubadoras en México y en América Latina; dicho modelo en el 2006 obtuvo su certificación como Modelo de Fomento Emprendedor (Poliemprende), lo que llevó a que la institución en el 2014 recibiera el Premio Nacional del Emprendedor. Bajo el anterior contexto, las preguntas de investigación son: 1) ¿Cuáles son las percepciones que tienen los estudiantes politécnicos con relación al fenómeno del emprendimiento en el instituto?, y 2) ¿Cuáles son los efectos principales de los modelos lineales generalizados de los factores de la ausencia de producto y modelo de negocio, la falta de apoyo institucional y los miedos para emprender con relación a las variables estudiadas?

El objetivo es analizar las percepciones que tienen los jóvenes politécnicos con relación al fenómeno del emprendimiento y los posibles obstáculos, como la ausencia de un modelo de negocio y producto, la falta de apoyo institucional y los miedos para emprender. La finalidad es identificar los avances y retrocesos que tienen las acciones desarrolladas por las escuelas, con el propósito de impulsar la educación emprendedora y promover en los estudiantes una mentalidad y espíritu emprendedor.

\section{El emprendimiento y la educación}

La evidencia reciente sugiere que existe una relación positiva entre el emprendimiento y la educación, ya que es en la universidad donde se adquieren muchas habilidades (Pisá-Bó, López-Muñoz y Novejarque-Civera, 2021). Es evidente que la educación para el emprendimiento coadyuva a la competitividad de los estudiantes, desarrolla las capacidades empresariales, genera habilidades que mejoran la personalidad y los talentos, estimula la creatividad e impulsa la autonomía (Guojin, 2011). Sumando a lo anterior, se afirma que el sistema educativo logra construir esa mentalidad emprendedora, además interviene para presentar el emprendimiento como alternativa posible para tener empleo de forma independiente. También es un hecho que en las instituciones de educación superior se forma la mayor parte de los futuros emprendedores, por lo que debe quedar claro que la futura educación empresarial tiene que dejar de pensar únicamente en enseñar conocimientos de cómo crear empresas y centrarse en experimentar el espíritu empresarial, además de enfocarse en la formación de ta- 
lentos con habilidades emprendedoras y de gestión (Raposo y Do Paço, 2011; Zeng, 2012; Liening, Geiger, Kriedel y Wagner, 2016; Vaquero-García, Ferreiro-Seoane y Álvarez-García, 2017).

El debate científico ha llevado a entender que la educación del espíritu empresarial no es solo la generación de las empresas sino también la capacidad que tienen los individuos para convertir ideas en acciones siendo más creativos y con mayor confianza en sí mismos (Kirby, 2018; Fallah Haghighi, Mahmoudi y Bijani, 2018). Se ha descubierto en jóvenes que han participado en programas de emprendimiento que un elemento clave para el desarrollo de actitudes emprendedoras está fuertemente asociado con el desarrollo de habilidades blandas como el incremento en el pensamiento creativo, saber tomar riesgos, resolver problemas y las aptitudes de liderazgo (Shahin, Ilic, Gonsalvez y Whittle, 2021).

La literatura ha resaltado el estudio de la perspectiva biológica en el espíritu empresarial desde la genética, la neurociencia, el neurodesarrollo y el comportamiento empresarial: se afirma que la biología no hace que las personas se conviertan en empresarios, lo que sí contribuye es la influencia que los individuos reciben en diferentes etapas de sus vidas con relación a variables que giran en torno al emprendimiento. Se tienen hallazgos importantes que indican que la testosterona está asociada con participar en actividades de emprendimiento; además, en materia de la neurociencia se ha encontrado que los vínculos afectivos de los emprendedores con sus nuevas empresas son similares a los vínculos que se tienen entre padres e hijos (Nicolaou, Phan y Stephan, 2021). Por último, el espíritu empresarial es considerado un mecanismo importante que coadyuva al desarrollo económico por medio de la generación de empleos, la innovación y el bienestar (Boubker, Arroud y Ouajdouni, 2021; Seelig, 2015). Por lo anterior, se espera que las universidades se involucren en aquellas actividades relacionadas con el espíritu empresarial tecnológico que tiene por objetivo resolver problemas nacionales (Kleine, 2020). Sin embargo, las soluciones que se esperan encontrar a las problemáticas de los países requieren enfocarse en la actitud empresarial (Acs, Szerb, Autio, 2016; Elert y Henrekson, 2021).

\section{Método}

\section{Participantes}

Los participantes en el estudio fueron estudiantes del Instituto Politécnico Nacional en los niveles de enseñanza media, superior y posgrado. Las características de los jóvenes fueron: a) estar inscritos en alguno de los tres niveles de enseñanza del IPN y b) contar con la disposición de responder el cuestionario. La muestra que se utilizó fue por conveniencia; se obtuvieron 9,626 instrumentos, de los cuales 5,028 pertenecen a la educación media superior, 4,149 educación superior y 449 al nivel de posgrado. Los cuestionarios se aplicaron en los meses de mayo a agosto del 2018. 


\section{Instrumento}

El cuestionario se estructuró con dos secciones, la primera integró 23 variables que permiten analizar el fenómeno del emprendimiento y la segunda parte incorporó 12 posibles obstáculos que impiden a los jóvenes emprender; la medición de estos se realizó con escalas del 1 al 12, se agruparon en tres factores. Todas las preguntas que se incorporaron en el instrumento fueron revisados y validados por expertos en el tema de emprendimiento. El enfoque de investigación utilizado es un estudio cuantitativo y transversal.

\section{Procedimiento}

Para facilitar la aplicación se realizó una invitación a las unidades académicas a través del Centro de Incubación de Empresas de Base Tecnológica (CIEBT); el cuestionario fue suministrado de forma electrónica. Se extendió la invitación a los 19 planteles de enseñanza media superior, 29 unidades de educación superior y 19 centros de investigación del Instituto Politécnico Nacional. La muestra que se alcanzó fue por la valiosa participación de las autoridades de las unidades académicas, que en algunos casos habilitaron salas de cómputo para incentivar a los estudiantes a que participaran en el estudio.

\section{Análisis de datos}

Se emplearon modelos lineales generalizados, ya que permiten identificar los efectos principales sobre las 23 variables de emprendimiento con relación a los tres factores que agruparon los obstáculos para emprender: 1) ausencia de producto y modelo de negocio, 2) falta de apoyo institucional y 3) miedos. Las veintitrés variables que son consideradas de entrada en el modelo fueron: 1) género, 2) área de conocimiento, 3) beca, 4) participación en proyectos vinculados, 5) capacitación para proyectos vinculados, 6) ingreso familiar, 7) se considera emprendedor, 8) la escuela promueve el emprendimiento, 9) participación en alguna actividad sobre emprendimiento en la unidad, 10) participación en actividades de emprendimiento fuera de la unidad, 11) tipos de proyectos, 12) la unidad académica brindó las herramientas para desarrollar el proyecto, 13) experiencia laboral fuera del IPN, 14) dónde se obtuvo la experiencia laboral, 15) cuenta con los conocimientos necesarios para administrar una empresa, 16) participación en algunos concursos de emprendimiento, 17) participación en concursos externos, 18) pensamiento sobre llevar a cabo algún emprendimiento en tres años, 19) interés por emprender, 20) tipos de fracaso, 21) han tenido algún negocio, 22) continúa la operación del negocio, 23) emprendimiento antes de ese negocio. También se hizo uso de las medias marginales que permiten ubicar, mediante los promedios obtenidos por cada categoría de las variables de entrada del modelo, los puntos máximos y mínimos otorgados por los participantes. Se llevó a cabo un 
estudio comparativo con los tres niveles de enseñanza, lo cual se logró trabajando con las pruebas de rachas para segmentar las bases de datos. Es importante mencionar que el modelo se dividió en dos partes para poder facilitar su análisis e interpretación.

\section{Resultados}

Se aplicaron 9,626 instrumentos, de los cuales 5,028 (52\%) pertenecen a la educación media, 4,149 (43\%) a la enseñanza superior y $449(5 \%)$ a posgrado. De forma general, el 58\% (5,652) son hombres y el 42\% $(3,974)$ mujeres. Las áreas de conocimiento de los participantes se presentan en la tabla 1.

Tabla 1. Participantes por área de conocimiento del Instituto Politécnico Nacional.

\begin{tabular}{|c|c|c|c|}
\hline Nivel educativo & Área de conocimiento & Participantes & Porcentaje \\
\hline \multirow[t]{7}{*}{ Educación media superior } & Ingeniería & 1,603 & $32 \%$ \\
\hline & Interdisciplinaria & 106 & $2 \%$ \\
\hline & Ciencias sociales & 812 & $16 \%$ \\
\hline & Ciencias de la salud & 653 & $14 \%$ \\
\hline & Físico matemáticas & 1,376 & $27 \%$ \\
\hline & Otro & 478 & $9 \%$ \\
\hline & Totales & 5,028 & $100 \%$ \\
\hline \multirow[t]{7}{*}{ Educación superior } & Ingeniería & 1,520 & $37 \%$ \\
\hline & Interdisciplinaria & 133 & $3 \%$ \\
\hline & Ciencias sociales & 692 & $17 \%$ \\
\hline & Ciencias de la salud & 677 & $16 \%$ \\
\hline & Físico matemáticas & 818 & $20 \%$ \\
\hline & Otro & 309 & $7 \%$ \\
\hline & Totales & 4,149 & $100 \%$ \\
\hline \multirow[t]{7}{*}{ Posgrado } & Ingeniería & 246 & $56 \%$ \\
\hline & Interdisciplinaria & 47 & $10 \%$ \\
\hline & Ciencias sociales & 38 & $8 \%$ \\
\hline & Ciencias de la salud & 64 & $14 \%$ \\
\hline & Físico matemáticas & 34 & $7 \%$ \\
\hline & Otro & 20 & $5 \%$ \\
\hline & Totales & 449 & $100 \%$ \\
\hline
\end{tabular}

Fuente: Construcción propia a partir de los resultados arrojados por el software SPSS.

En la investigación se les cuestionó sobre si habían participado en algún proyecto vinculado del IPN: se halló que únicamente el $20 \%$ de los jóvenes de enseñanza media superior (EMS) señalaron que sí; por su parte, el 22\% de los alumnos de educación superior (ES) han estado en ese tipo de proyectos, y el 21\% de los estudiantes de 
posgrado se han involucrado en proyectos del IPN. También se les preguntó sobre si habían recibido capacitación para su participación en los proyectos vinculados, los resultados son desalentadores: solo el 15\% de la población de educación media enfatizó que sí; en el caso de la muestra de educación superior, el 16\% afirmó haber recibido esa capacitación, y el 15\% del estrato de enseñanza en posgrado indicó que le habían brindado la instrucción.

El estudio indagó sobre si los participantes se consideraban emprendedores. Se encontró que el 67\% de la población de educación media dijo que sí; de igual forma, el 67\% de los alumnos de enseñanza superior enfatizó sentirse emprendedores, y el $72 \%$ del estrato correspondiente a los jóvenes de posgrado señaló percibirse como emprendedores. Otro hallazgo importante es que el 67\% de los estudiantes de educación media expresó que su unidad académica promueve el emprendimiento; por su parte el 65\% de la muestra de las escuelas de enseñanza superior estuvo de acuerdo en que en su escuela se impulsa el emprendimiento; por último, $62 \%$ de los alumnos de posgrado de igual forma dijo que su centro fomenta el emprendimiento. Sin embargo, cuando se les cuestiona sobre si ellos han participado en actividades de emprendimiento es sus escuelas se halló que solo el 15\% de los encuestados de educación media dijo que sí; de igual manera solo el 15\% de alumnos universitarios se ha involucrado en ese tipo de actividades, aunado al 11\% de la población de posgrado que indicó haber estado en ese tipo de actividades.

También se investigó sobre si los estudiantes habían participado en alguna actividad de emprendimiento fuera de la unidad académica: los resultados indicaron que únicamente el $20 \%$ de los alumnos de educación media había estado en este tipo de actividades; el 20\% de los estudiantes de educación superior expresó haber estado en ese tipo eventos, y el 23\% del estrato de la comunidad de posgrado dijo haber tenido esa oportunidad.

Por otra parte, en la investigación se les cuestionó a los estudiantes si su proyecto de prototipo, tesis o trabajo final surge a partir de: a) experiencia propia, b) proyecto de investigación, c) proyecto de tesis, d) sugerencia del profesor y e) otros. Los resultados reflejaron que en el caso del estrato de jóvenes de educación media el 32\% de los proyectos surge de proyectos de investigación, de igual forma el 32\% de los jóvenes de enseñanza superior, y en el caso de la población de posgrado fue del 31\%. También se encontró en el 22\% de los alumnos de bachillerato que los proyectos tienen su origen de experiencia propia; por su parte el $20 \%$ de los estratos de enseñanza superior y posgrado recurre a ideas que los estudiantes han generado durante sus experiencias de vida. De igual forma se halló que el 7\% de los participantes de preparatoria y de superior generó sus proyectos a partir de sus investigaciones de tesis, y el 8\% en posgrado inicia de igual forma ese tipo de indagaciones. Un dato que se encontró es que el $9 \%$ de los estudiantes encuestados en todos los niveles dijo 
que los proyectos se han desarrollado a partir de las sugerencias de algún profesor. Por último, se encontró que el 30\% de los grupos conformados por los alumnos en educación media y superior enfatizó que sus proyectos iniciaron de otros elementos; por su parte, el 32\% de los estudiantes de posgrados señaló de igual forma que son otros los factores que influyeron en el surgimiento de sus proyectos.

A los participantes se les preguntó respecto a si las unidades académicas les habían proporcionado las herramientas para emprender el proyecto. Los hallazgos que se obtuvieron resultan interesantes: el 61\% de los jóvenes de enseñanza media afirmó que sí, mientras el 39\% dijo que no; por otro lado, el 59\% de los encuestados de educación superior señaló que su unidad académica le había dado las herramientas para trabajar el proyecto, mientras que el $41 \%$ dijo que no; en el caso del grupo de los alumnos de enseñanza de posgrado, el 58\% dijo que sí al tema de las herramientas de emprendimiento y el $42 \%$ que no.

A los jóvenes se les cuestionó si contaban con experiencia laboral fuera del Instituto Politécnico Nacional: el 41\% de los alumnos de nivel bachillerato y de enseñanza superior dijo que sí, el 59\% que no; por su parte, el 44\% de los estudiantes de posgrado afirmó que sí y el 56\% indicó que no. Ahora bien, se les preguntó a los participantes en qué área habían tenido la experiencia laboral y se halló que el 8\% de los jóvenes de preparatoria y de educación superior tenía experiencia operativa en empresas; en el caso de los encuestados de nivel posgrado, solo el 9\%. Además, el 5\% de los alumnos de las muestras de enseñanza media y superior indicó haber estado como empleados de dependencias públicas, mientras que el 6\% de la población de posgrado aseveró haber estado en alguna dependencia del sector público. Se encontró que solo el 1\% de los universitarios habían sido consultores, y el $2 \%$ en el caso de los alumnos de posgrado. Por su parte, el 3\% de los participantes de preparatoria y universitarios afirmó haber estado trabajando como docentes; en el caso del grupo de posgrado se obtuvo un 5\%. Otro hallazgo es que únicamente el $2 \%$ de los estudiantes de bachillerato y de educación superior expresó haber tenido experiencia gerencial en empresas, mientras el 3\% de alumnos de posgrado ha obtenido ese tipo de empleo. De igual forma se encontró que solo el 3\% de los encuestados de todos los niveles indicó haber tenido un empleo relacionado con la investigación básica. Además se identificó que el 1\% de los estudiantes de posgrado dijo haber tenido experiencia en el ámbito de los desarrollos tecnológicos; por otro lado, se descubrió que el 2\% de los encuestados de enseñanza media y superior enfatizó haber tenido ese tipo de experiencias. Por último, el 55\% de los involucrados en el estudio de bachillerato enfatizó que otras han sido sus experiencias laborales; por su parte, el 55\% de los universitarios consideró que sus vivencias laborales provienen de otros elementos y el 50\% de los entrevistados de posgrado dijo que sus antecedentes laborales tienen su origen en otros sectores. 
En el estudio se encontró que el 42\% de los participantes universitarios y de posgrado dijo que cuentan con los conocimientos y habilidades necesarios para administrar y gestionar una empresa, pero el 58\% dijo que no. En el caso de los alumnos de bachillerato, el $43 \%$ afirmó contar con ese tipo de conocimientos, mientras que el 57\% enfatizó que no.

Por otra parte se encontró que solo el $6 \%$ de los estudiantes de preparatoria y universitarios indicó que han participado en algún concurso de emprendimiento institucional; por su lado, el 7\% de los alumnos de posgrado señaló que han concursado en temas de emprendimiento. A los participantes se les preguntó si habían estado en algún concurso de emprendimiento externo: los hallazgos revelaron que el 5\% de los participantes de bachillerato y educación superior ha estado presente en concursos externos al instituto, en el caso de la muestra de posgrado únicamente el 7\%.

Se tiene como hallazgo relevante que el $89 \%$ de los estudiantes de bachillerato y nivel superior enfatizó que está interesado en realizar algún emprendimiento en los próximos tres años. Por su parte, el 92\% de los participantes del posgrado señaló que desea llevar a cabo el emprendimiento. Cuando se les cuestionó por qué les interesa emprender, el 35\% de los alumnos de preparatoria y nivel superior dijo que es por contar con independencia económica; en el caso de la muestra de posgrado, el 33\% anhela esta. También se halló que el 18\% de los alumnos de nivel medio superior y universidad indicó que le gusta la idea de iniciar un negocio por el tema del autoempleo, mientras que $20 \%$ de los participantes de posgrado enfatiza que tener un empleo propio es lo que les genera interés para emprender. Además, el 14\% de los niveles de bachillerato y educación superior dijo que la atracción por emprender se debe a la oportunidad tecnológica; de igual forma el 19\% de los participantes de posgrado detecta la coyuntura tecnológica. Un hallazgo que se obtuvo es que solo el 8\% de los entrevistados de enseñanza media, el 9\% de universitarios y el 7\% de posgrado indicaron que buscan emprender para complementar el gasto familiar. Por último, el 10\% de la muestra de preparatoria y el 10\% de educación superior está seguro de que quiere emprender por la oportunidad que se presenta en el mercado, y el 11\% de los jóvenes de posgrado desea de igual forma emprender para poder explotar este. El resto de los encuestados considera que son otros los factores que intervienen para desarrollar su interés por emprender.

En el estudio se descubrió que el 48\% de los encuestados de educación media y superior señaló que para ellos el fracaso es aprendizaje; de igual forma el 49\% de los alumnos de posgrado estuvo de acuerdo. Además, el 40\% de los participantes en todos los niveles considera que el fracaso es una oportunidad de aprendizaje. Por último, el 12\% de los estudiantes de educación media piensa que el fracaso representa un error; por su parte, el 12\% de los encuestados de nivel universitario de igual forma opina que los fracasos son errores en la vida, y el 11\% de los estudiantes de posgrado tiene esa apreciación. 
A los encuestados se les cuestionó sobre si habían tenido algún negocio; el 25\% de los participantes de bachillerato y educación superior indicaron que sí y el 75\% dijeron que no, mientras que el 32\% de los jóvenes de posgrado aseveró haber contado con algún negocio y el 68\% estableció que no. Posteriormente se les preguntó sobre si continuaba operando el negocio, se descubrió que únicamente en el 11\% de los jóvenes de educación media y de educación superior el emprendimiento continuaba en el mercado.

A los jóvenes se les preguntó sobre las apreciaciones acerca de los posibles obstáculos que les impiden emprender, considerando una puntuación del 1 al 12, siendo el número 12 el más alto en la percepción. En la figura 1 y en la tabla 2 se muestran los resultados. Se halló que los principales obstáculos en los tres niveles de enseñanza fueron cuatro: 1) no tiene producto definido, 2) falta de modelo de negocio, 3) falta de recursos económicos y 4) falta de equipo de trabajo.

\section{Modelo lineal generalizado de la dimensión 1.}

\section{Ausencia de producto y modelo de negocio}

En esta dimensión se analizan las apreciaciones de los jóvenes respecto a los obstáculos para emprender relacionados con la ausencia de producto, falta de modelo de negocio, desconocimiento de los competidores y escasos conocimientos para emprender. Se aplicó el modelo lineal generalizado multivariante; con respecto a la primera parte del

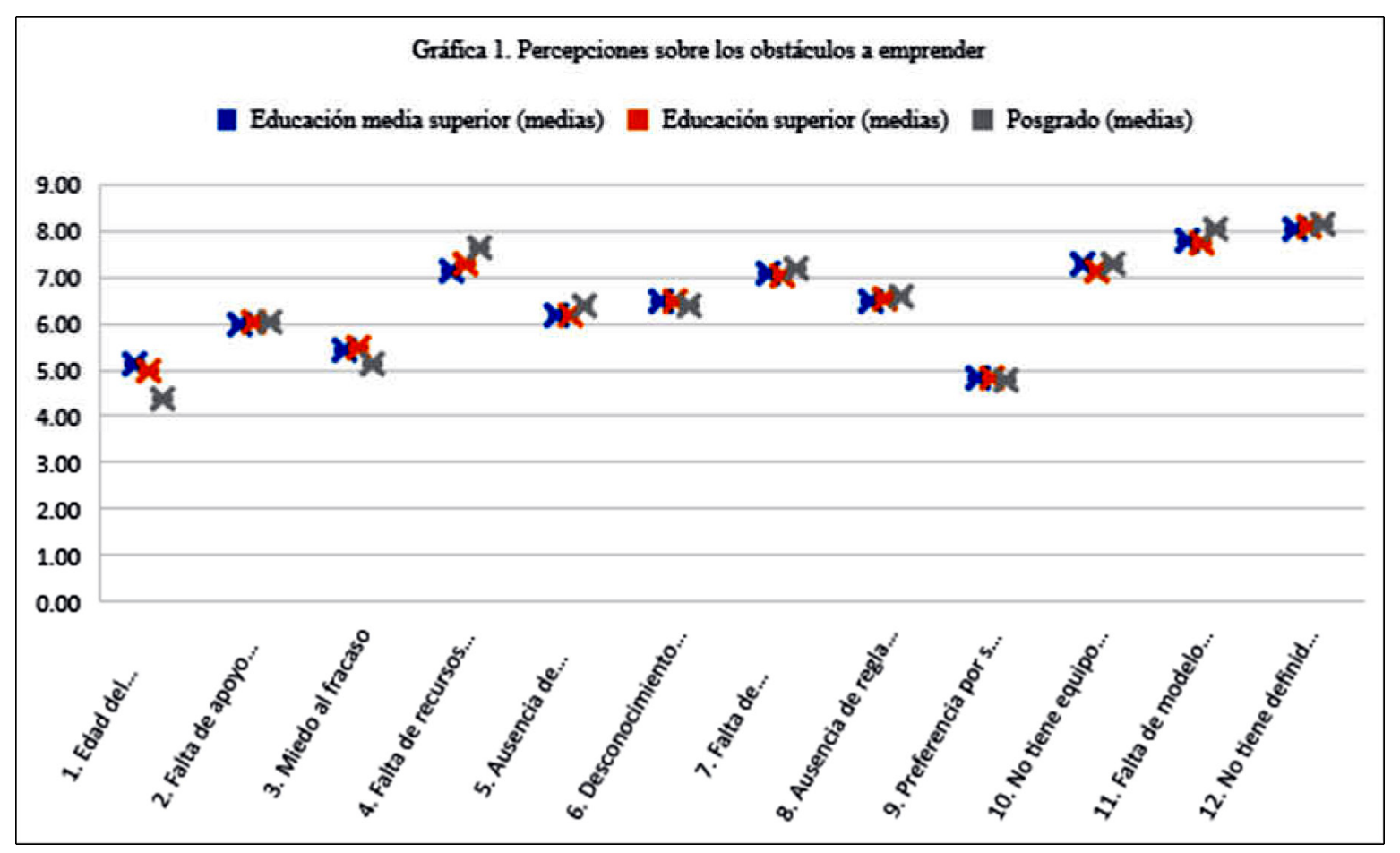

Figura 1. Percepciónes sobre los obstáculos a emprender.

Fuente: Construcción propia a partir de los resultados obtenidos del SPSS. 
Tabla 2. Apreciaciones sobre los impedimentos para emprender de los participantes.

Variables sobre los impedimentos para emprender

1. Edad del emprendedor

2. Falta de apoyo público

3. Miedo al fracaso

4. Falta de recursos económicos

5. Ausencia de respaldo institucional

6. Desconocimiento de los emprendedores

7. Falta de conocimientos para emprender

8. Ausencia de reglas o normatividad

9. Prefiero ser empleado

10. No tiene equipo de trabajo

11. Falta de modelo de negocio

12. No tiene definido un producto
Educación media superior (medias)
Educación superior (medias)
Posgrado (medias)

\begin{tabular}{lll}
5.15 & 5.00 & 4.35 \\
\hline 5.98 & 6.03 & 6.03 \\
\hline 5.42 & 5.47 & 5.13 \\
\hline 7.15 & 7.28 & 7.65 \\
\hline 6.18 & 6.19 & 6.37 \\
\hline 6.47 & 6.50 & 6.40 \\
\hline 7.07 & 7.06 & 7.17 \\
\hline 6.46 & 6.52 & 6.58 \\
\hline 4.81 & 4.83 & 4.79 \\
\hline 7.27 & 7.12 & 7.31 \\
\hline 7.80 & 7.74 & 8.03 \\
\hline 8.03 & 8.10 & 8.15
\end{tabular}

Fuente: Construcción propia a partir de los resultados del SPSS.

Tabla 3. Prueba de contraste de ómnibus de cada dimensión analizada.

Factor 1. Ausencia de producto y modelo de negocio

Chi-cuadrado de razón
Factor 2. Falta de apoyo institucional Chi-cuadrado de razón de verosimilitud de verosimilitud

p

Factor 3. Miedos para emprender

Chi-cuadrado de razón de verosimilitud

Parte 1 del modelo

\begin{tabular}{|c|c|c|c|c|c|c|}
\hline Educación media superior & 67.907 & $0.000^{* *}$ & 102.897 & $0.000^{* *}$ & 81.007 & $0.000^{* *}$ \\
\hline Educación superior & 51,749 & $0.008^{* *}$ & 72.862 & $0.000^{* *}$ & 65.865 & $0.000^{* *}$ \\
\hline Posgrado & 48.261 & $0.001 * *$ & 30.394 & 0.446 & 39.016 & 0.125 \\
\hline \multicolumn{7}{|c|}{ Parte dos del modelo } \\
\hline Educación media superior & 31.957 & $0.010^{*}$ & 38.334 & $0.001 * *$ & 48.932 & $0.000 * *$ \\
\hline Educación superior & 15.118 & 0.516 & 35.656 & $0.003^{* *}$ & 28.162 & $0.030 * *$ \\
\hline Posgrado & 27.351 & 0.038 & 11.705 & 0.764 & 25.742 & 0.058 \\
\hline
\end{tabular}

${ }^{*} p<0.05,{ }^{* *} p<0.01$.

Fuente: Construcción propia a partir de los resultados del SPSS.

modelo se hallaron efectos en educación media superior en las variables de ingreso familiar $(p=0.01<0.05)$, la escuela promueve el emprendimiento $(\phi=0.03<0.05)$, cuento con los conocimientos necesarios para administrar una empresa $(\phi=0.00<$ 0.05) (ver tabla 4). El análisis de las medias mostró que los jóvenes de bachillerato cuyas familias tienen un ingreso mensual aproximado de 60,000 pesos son los que califican con más puntos la dimensión de la ausencia de un modelo de negocio y producto. También se halló que los jóvenes que consideran que su unidad académica 
promueve el emprendimiento son los que consideran que la falta del producto y del modelo del negocio es una de sus principales barreras para emprender. Sin embargo, se encontró que los alumnos que indicaron que no cuentan con los conocimientos y habilidades necesarias para gestionar una empresa son aquellos que puntean más alto que la falta del modelo de negocio y producto es lo que los detiene a emprender.

En el caso del nivel de educación superior se ubicó el efecto del modelo en la variable "cuento con los conocimientos y habilidades para gestionar una empresa" $(\phi=0.04<0.05)$ (ver tabla 4$)$. Se encontró que aquellos estudiantes que otorgaron más puntos son quienes piensan que no cuentan con las habilidades para administrar una empresa y por ende no emprenden.

En el estrato de los posgrados, las variables con consecuencias en el modelo fueron en la participación de proyectos vinculados $(p=0.02<0.05)$, experiencia laboral fuera del instituto $(p=0.00<0.05)$ y contar con los conocimientos para gestionar una empresa $(\phi=0.00<0.05)$ (ver tabla 4). Se halló que los jóvenes que dijeron haber participado en algún proyecto vinculado son los que le dieron más puntos a la falta de producto y modelo de negocio que ha provocado que no decidan emprender. Ahora bien, los jóvenes que dijeron que no tienen experiencia laboral fuera del instituto son los que califican más alto el obstáculo relacionado con la ausencia de productos y del modelo de negocio. Otro hallazgo es que los estudiantes que mencionan que perciben que no tienen los conocimientos para gestionar una empresa son los que califican con más puntos la barrera de la carencia del producto y del modelo de negocio.

En la parte dos del modelo los efectos se tienen en el grupo de enseñanza media en las variables de la participación en concursos externos $(p=0.03<0.05)$, el interés en realizar emprendimiento en los próximos tres años $(p=0.04<0.05)$ y si continua la operación del negocio que en su momento los jóvenes emprendieron en el pasado $(\phi=0.05=<0.05)$ (ver tabla 4). Se encontró que los alumnos de enseñanza media que indicaron que no han participado en concursos de emprendimiento externo perciben con mayor intensidad la falta de modelos de negocios y producto como inconveniente para poder emprender. Además, los jóvenes que afirmaron que no les interesa realizar algún emprendimiento en los próximos tres años son los que sienten con mayor prevalencia la escasa presencia del modelo de negocio. Por último, el grupo de participantes que dijeron que el negocio que habían emprendido ya no está operando son los que piensan que la ausencia del esquema de modelo y no tener un producto es lo que les impide volver a emprender.

En la parte dos del modelo se identificaron efectos en el grupo de los participantes de posgrado, hallándose las variaciones en la participación en algún concurso de emprendimiento institucional $(p=0.00<0.05)$ y la intención de realizar algún emprendimiento en los próximos tres años $(p=0.00<0.05)$ (ver tabla 4). Se halló que los alumnos de posgrado que no han participado en algún concurso del instituto son los que sienten como barrera la ausencia del modelo de negocio. También se 
Tabla 4. Modelos lineales generalizados por factor.

\begin{tabular}{|c|c|c|c|c|c|c|c|c|c|c|}
\hline \multirow[b]{2}{*}{ Partes } & \multirow[b]{2}{*}{ Niveles educativos } & \multicolumn{3}{|c|}{$\begin{array}{l}\text { Ausencia de producto y } \\
\text { modelo de negocios }\end{array}$} & \multicolumn{3}{|c|}{$\begin{array}{c}\text { Falta de } \\
\text { apoyo institucional }\end{array}$} & \multicolumn{3}{|c|}{ Miedos } \\
\hline & & EMS & ES & Posgrado & EMS & ES & Posgrado & $\overline{\text { EMS }}$ & ES & $\overline{\text { Posgrado }}$ \\
\hline \multirow{15}{*}{$\begin{array}{l}\text { Parte } 1 \\
\text { del } \\
\text { modelo }\end{array}$} & Género & .971 & .161 & .594 & .002 & .466 & .245 & $.001 *$ & $.018^{*}$ & .622 \\
\hline & Área de estudio & .191 & .081 & .835 & .079 & .480 & .889 & $.000 * *$ & $.005^{*}$ & .605 \\
\hline & $\underline{B e c a}$ & .510 & .652 & .801 & $.045^{*}$ & .670 & .911 & .212 & .475 & .860 \\
\hline & Participación en proyectos vinculados & .820 & .205 & $.028 *$ & .325 & $.044^{*}$ & .734 & .535 & .822 & .037 \\
\hline & Capacitación para proyectos vinculados & .525 & .808 & .412 & .558 & .231 & .900 & .920 & .509 & .437 \\
\hline & Ingreso familiar & $.012 *$ & .095 & .815 & .142 & .828 & .551 & $.046^{*}$ & .088 & .985 \\
\hline & Se considera emprendedor & .261 & .379 & .689 & $.018^{*}$ & .820 & .633 & .423 & .741 & .337 \\
\hline & La escuela promueve el emprendimiento & $.031 *$ & .069 & .128 & $.000^{* *}$ & $.000^{* *}$ & .107 & .229 & .251 & .853 \\
\hline & $\begin{array}{l}\text { Participación en alguna actividad sobre } \\
\text { emprendimiento en la unidad }\end{array}$ & .781 & .207 & .445 & .871 & $.001^{*}$ & .413 & .837 & .093 & .111 \\
\hline & $\begin{array}{l}\text { Participación en actividades de } \\
\text { emprendimiento fuera de la unidad }\end{array}$ & .133 & .211 & .440 & .245 & .089 & .444 & .936 & .629 & .862 \\
\hline & $\begin{array}{l}\text { Proyectos de prototipos, tesis, trabajo final } \\
\text { surgen de tesis, etc. }\end{array}$ & .266 & .163 & $.032 *$ & .258 & .202 & .215 & .182 & .384 & .174 \\
\hline & $\begin{array}{l}\text { La unidad académica brindó las herramientas } \\
\text { para desarrollar el proyecto }\end{array}$ & .365 & .973 & .567 & .879 & $.027 *$ & .634 & .589 & $.020 *$ & .248 \\
\hline & Experiencia laboral fuera del IPN & .707 & .586 & $.003^{*}$ & .779 & .990 & .099 & .616 & .579 & .081 \\
\hline & Dónde la experiencia laboral & .146 & .342 & .097 & .510 & .464 & .466 & .090 & .197 & .028 \\
\hline & $\begin{array}{l}\text { Cuento con los conocimientos necesarios } \\
\text { para administrar una empresa }\end{array}$ & $.000 * *$ & $.049 *$ & $.000^{* *}$ & $.000^{* *}$ & .196 & .062 & .474 & .249 & .022 \\
\hline \multirow{8}{*}{$\begin{array}{l}\text { Parte } 2 \\
\text { del } \\
\text { modelo }\end{array}$} & $\begin{array}{l}\text { Participación en algún concurso de } \\
\text { emprendimiento institucional }\end{array}$ & .573 & .996 & $.004 *$ & .839 & .451 & .395 & .767 & .519 & $.011^{*}$ \\
\hline & Participación en algún concurso externo & .033 & .159 & .835 & .173 & .231 & .965 & .374 & .626 & .846 \\
\hline & $\begin{array}{l}\text { Realizar emprendimiento en los próximos } \\
\text { tres años }\end{array}$ & $.046^{*}$ & .286 & $.001 * *$ & .302 & .971 & .276 & $.006^{*}$ & .174 & $.009 * *$ \\
\hline & Interés por emprender & .570 & .977 & .441 & .409 & .104 & .277 & $.015^{*}$ & .145 & .486 \\
\hline & Tipos de fracaso & .302 & .981 & .396 & .140 & .714 & .678 & $.001 * *$ & .801 & .759 \\
\hline & Ha tenido algún negocio & .157 & .104 & .329 & .154 & .586 & .891 & .755 & $.023 *$ & .329 \\
\hline & Continúa la operación del negocio & $.050 *$ & .525 & .360 & $.009 *$ & $.028 *$ & .775 & .702 & $.010^{*}$ & .395 \\
\hline & Emprendimiento antes de ese negocio & .581 & .226 & .747 & .888 & $.006^{* *}$ & .555 & .276 & .079 & .367 \\
\hline
\end{tabular}

$* p<0.05, * * p<0.01$

Fuente: Construcción propia a partir de los resultados del SPSS.

identificó que los jóvenes que señalaron que no tienen interés en realizar algún emprendimiento en los próximos tres años son los que dieron más puntos a la ausencia de los modelos de negocios como obstáculo para emprender.

Modelo lineal generalizado de la dimensión 2.

Falta de apoyo institucional

En la dimensión 2 se mide la percepción sobre los obstáculos que impiden a los estudiantes emprender, relacionados con la ausencia de apoyo público, la carencia del respaldo institucional, la falta de reglas y la preferencia que se tiene por ser empleado. 
En la primera parte del modelo se encontraron efectos en el grupo de los jóvenes de bachillerato en las variables de las becas $(p=0.04<0.05)$, si se considera emprendedor $(p=0.01<0.05)$, si la escuela promueve el emprendimiento $(p=0.00<0,05)$ y contar con los conocimientos necesarios para administrar una organización $(p=0.00$ $<0.05$ ) (ver tabla 4). Se halló que el grupo de jóvenes que indicaron que tenían una beca son los que dieron más puntos a la falta de apoyo institucional para emprender. Asimismo, los alumnos que dijeron que ellos se consideran emprendedores de igual manera perciben la carencia de apoyo como una barrera para emprender. Hay otro hallazgo en el grupo de participantes que señalaron que la unidad académica no promueve el emprendimiento: son quienes de igual forma resaltan como obstáculo la falta de apoyo para emprender. En contraste, los alumnos que califican con mayor cantidad de puntos la falta de apoyo son aquellos participantes que afirmaron que tienen los conocimientos necesarios para gestionar una empresa.

En el grupo de los jóvenes de enseñanza superior se encontraron efectos en el modelo en las variables de: a) la participación en proyectos vinculados $(p=0.04<$ $0.05)$, b) si la escuela promueve el emprendimiento $(p=0.00<0.05)$, c) participación en alguna actividad de emprendimiento en la unidad $(p=0.00<0.05)$ y d) si la unidad académica brinda herramientas para desarrollar el proyecto $(\phi=0.02<0.05)$ (ver tabla 4). Los hallazgos indican que los universitarios que habían participado en proyectos vinculados en el IPN percibieron en mayor medida el escaso apoyo público; además, los alumnos que señalaron que la escuela no promueve el emprendimiento dieron mayores puntos a la falta de apoyo como elemento que detiene su interés por emprender. En contraste, la muestra de jóvenes que enfatizaron que habían participado en alguna actividad emprendimiento son aquellos que sienten con mayor intensidad la ausencia de apoyo. Por último, el grupo de estudiantes que dijeron que la escuela no les había otorgado las herramientas necesarias para llevar a cabo su proyecto de igual manera son los que reafirman el sentimiento del poco apoyo institucional y público.

En la parte dos del modelo que mide las variables de 1) participación en concursos de emprendimiento institucional, 2) participación en concursos de emprendimiento externo, 3) interés por realizar algún emprendimiento en los próximos tres años, 4) por qué les interesa emprender, 5) para el participante qué significa el fracaso, 6) saber si ha tenido algún negocio, 7) saber si sigue en funcionamiento el negocio y 8) identificar si había emprendido antes, se obtuvo que los efectos principales se detectaron en los grupos de los jóvenes de educación media y en los universitarios. En el caso de la enseñanza media se ubicaron los impactos en la variable "el negocio que emprendiste continúa operando" ( $p=0.00<0.05$ ) (ver tabla 4 ), en la cual se encontró que los jóvenes que sienten la carencia de apoyo son los que afirman que sus negocios continúan operando.

Los efectos en el modelo multivariante de enseñanza superior se ubicaron en a) continúa en operación en negocio que emprendiste $(p=0.02<0.05)$ y b) habías 
emprendido antes $(p=0.00<0.05)$ (ver tabla 4). Los hallazgos señalan que los universitarios que dijeron que su negocio sigue en operación son los que consideran la inexistencia de apoyo. Por otra parte, los jóvenes que afirmaron que anteriormente habían emprendido son los que estimaron la ausencia de apoyo.

\section{Modelo lineal generalizado de la dimensión 3.}

\section{Los miedos para emprender}

En esta dimensión se analizan las percepciones que tienen los jóvenes con relación a los miedos, como lo representa el miedo al fracaso, la edad del emprendedor y no contar con un equipo para trabajar. En la primera parte del modelo los efectos en el caso de los estudiantes de enseñanza media se hallaron en el género $(p=0.01<0.05)$, el área de estudio $(p=0.00<0.05)$ y el ingreso familiar $(p=0.04<0.05)$ (ver tabla 4). Se halló que las mujeres son las que perciben en mayor medida los miedos para emprender; por otra parte, se identificó que los alumnos del área interdisciplinaria son los que sienten con mayor intensidad los miedos, y los que menos son los estudiantes del área de las Ciencias de la salud. También los participantes que afianzaron más sus miedos son aquellos que afirmaron que su familia tiene un ingreso menor de 10,000 pesos mensuales.

En el estudio se hallaron efectos en el modelo de los jóvenes de educación universitaria en las variables del género $(p=0.01<0.05)$, área de estudio $(p=0.00<0.05)$ y la unidad académica brindó las herramientas para desarrollar el proyecto $(p=0.02<$ $0.05)$ (ver tabla 4). Se encontró que los miedos son en mayor medida percibidos por las mujeres. Además, los participantes que calificaron más alto los miedos fueron los del área de Físico matemáticas, y los que menos sienten los miedos fueron los participantes del área de Ciencias de la salud. De igual forma, los jóvenes que indicaron que su unidad académica les había brindado las herramientas para el desarrollo de sus proyectos son los que sintieron con mayor intensidad los obstáculos para emprender.

En la parte dos del modelo se hallaron variaciones en los tres niveles educativos. En el caso de los estudiantes de educación media superior, los efectos del modelo se ubicaron en las variables de intención de realizar algún emprendimiento en los próximos tres años $(p=0.00<0.05)$, los intereses por emprender $(p=0.01<0.05)$ y la percepción que tienen del fracaso $(\phi=0.00<0.05)$ (ver tabla 4). Se encontró que los participantes que sienten más los temores por emprender son aquellos que afirmaron que les interesa realizar algún emprendimiento en los próximos tres años. Otro de los hallazgos que se obtuvieron es que aquellos jóvenes que dijeron que les interesa emprender por tradición familiar son los que sienten con más fuerza los miedos para llevar a cabo el emprendimiento, y los que calificaron con menos puntos son aquellos que indicaron que les interesa emprender por complementar el ingreso y por las oportunidades de mercado. Por último, se identificó que los jóvenes que 
sienten mucho más miedo son aquellos que afirmaron que para ellos el fracaso es igual a los errores.

En el caso de los estudiantes de educación superior las variaciones de los efectos se ubicaron en si han tenido algún negocio $(p=0.02<0.05)$ y si continúa la operación del negocio $(p=0.01<0.05)$ (ver tabla 4$)$. Se descubrió que los universitarios que dijeron que han tenido algún negocio calificaron más alto los temores por emprender. Además, los jóvenes que indicaron que sus negocios ya no están operando son los que piensan en los temores para emprender.

Por último, con respecto a los jóvenes que estudian posgrado, se hallaron variaciones en las variables de la participación en algún concurso de emprendimiento institucional $(p=0.00<0.05)$ e interés en realizar algún emprendimiento en los próximos tres años $(p=0.00<0.05)$ (ver tabla 4 ). El estudio arrojó que las jóvenes que señalaron haber participado en algún concurso institucional son las que dan mayores puntos a los miedos que obstaculizan emprender. Por último, los estudiantes que expresaron su interés por llevar a cabo algún emprendimiento dentro de los siguientes tres años son los que en su mayoría calificaron más alto los miedos.

\section{Discusión}

En la investigación se hallaron evidencias que coinciden con estudios previos realizados por Pérez, Núñez y Montoya (2020), que sostienen que los estudiantes politécnicos en general muestran un bajo interés por crear empresas: se encontró que únicamente el 25\% de jóvenes de enseñanza media y superior, y el 32\% de posgrado, dijeron haber tenido algún negocio; lamentablemente, solo el 11\% de los estudiantes de la población de bachillerato y superior mencionaron que el emprendimiento sigue en operación. A pesar de los resultados desalentadores en materia de negocios generados en el transitar de la carrera de los jóvenes, se encontró un resultado esperanzador que señala que el 89\% de los estudiantes de enseñanza media y superior están interesados en llevar a cabo algún emprendimiento en los próximos tres años; por su parte el 92\% de los participantes de posgrado dijo lo mismo. Estos datos reflejan que los estudiantes han pensado en emprender, por lo que es necesario trabajar en acciones que les ayuden a obtener una educación emprendedora capaz de apoyar sus iniciativas (Egerová, Eger y Mièík, 2017; Fallah, Mahmoudi y Bijani, 2018).

Sin embargo, siguiendo con los resultados, se encontró que únicamente un promedio del 15\% de jóvenes de enseñanza media y superior habían participado en algunas actividades de emprendimiento en sus escuelas; en el caso de la población de posgrado solo el 11\%. Estos datos reflejan que los jóvenes posiblemente muestren desinterés porque no han desarrollado habilidades emprendedoras, las cuales requieren generar ideas, para lo cual se necesita trabajar las competencias creativas (Mróz y Ocetkiewicz, 2021). 
En contraste se tienen resultados alentadores derivados de la investigación que muestran que el $67 \%$ de jóvenes de educación media y superior se sienten emprendedores y el $72 \%$ afirmaron de igual forma sentirse de esa forma: esto indica que se puede trabajar en generar y fortalecer la mentalidad de los estudiantes orientada al emprendimiento. Estos resultados son consistentes con lo expuesto por Ndou, Secundo y Mele (2016). Rivero y Ubierna (2021) afirman que se puede crear empresarios equipando a los jóvenes de una mentalidad emprendedora; se tiene que insistir en que, para poder crear ideas, las instituciones tienen que contemplar el desarrollo de la creatividad (Anjum, Farrukh, Heidler y Díaz, 2021).

Por otra parte, en la investigación se analizaron tres factores que miden la percepción de los obstáculos para emprender de parte de los jóvenes: 1) la ausencia de producto y modelo de negocio, 2) la falta de apoyo institucional y 3) los miedos para emprender. Con respecto al factor que mide la ausencia de producto y modelo de negocio, los hallazgos de los modelos lineales generalizados reflejan que, en los tres niveles educativos, la percepción de la falta de conocimiento en la gestión de negocios sigue siendo un impedimento para llevar a cabo el emprendimiento. Se tiene que destacar que el temor para emprender por parte de los estudiantes de posgrado es por su carencia de experiencia laboral.

Ahora bien, los resultados que señalan la falta de conocimiento de gestión que no permite tener la claridad en el diseño del producto y el modelo de negocio; estos resultados refuerzan lo explicado por Raposo y do Paço (2011), quienes mencionan que la enseñanza empresarial tiene que dotar a los estudiantes de conocimientos de gestión, pero además es necesario que se les proporcionen experiencias reales que les permitan vivir el emprendimiento y desarrollar capacidades para enfrentar el éxito y el fracaso. También se debe tener cuidado con las acciones para fomentar la educación empresarial en los jóvenes, pues los resultados en ocasiones tienen efectos débiles, debido a que depende de la percepción que tienen los estudiantes con relación a los cursos y actividades extracurriculares. Además es positivo que los emprendedores reciban mentoría y coaching que los apoye en los momentos más complicados (Sáfrányné y Bartha, 2021; Games, 2021).

En el factor que mide los obstáculos por emprender por la falta de apoyo del sector público se encontraron efectos en los jóvenes de educación media que dijeron considerarse emprendedores, que piensan que cuentan con conocimientos de gestión y que afirmaron que sus escuelas no promueven el emprendimiento. En contraste, los resultados de la población de educación superior cambian en el sentido de que la ausencia de apoyo institucional se percibe con mayor fuerza en aquellos estudiantes que sí han participado en proyectos vinculados, además de haberse involucrado en actividades de emprendimiento, aunado a la percepción que tienen de las escasas herramientas que las escuelas les han proporcionado en el momento de llevar a cabo 
el emprendimiento; por ello reafirman sus ideas de que las escuelas no promueve el emprendimiento. Estudios previos han señalado que no todos los emprendedores son iguales y la realidad es que muy pocos logran crecer sus negocios de manera que permita generar puestos de trabajo y de esa forma aportar a la economía de las naciones (Acs, Szerb y Autio, 2016; Dionisio, Júnior y Fischer, 2021).

En el factor tres, que mide el miedo al fracaso, se halló que las mujeres de enseñanza media y superior son las que perciben con mayor fuerza los miedos por emprender, por el temor fracasar. Además, se reafirman estos miedos en aquellos jóvenes con familias de bajos ingresos económicos. Considerando los resultados encontrados, se sabe que el impacto de la educación empresarial es sensible al género y a la edad; también hay un impacto positivo para los varones, pero no pasa lo mismo con las mujeres, ya que, para ellas, se tiene un resultado negativo en sus intenciones emprendedoras (Brüne y Lutz, 2020; Hung, 2020; Koyuncuoglu, 2021; Nicolaou, Phan y Stephan, 2021). Los resultados encontrados contribuyen a los hallados por Wyrwich, Stuetzer y Sternberg (2016), quienes afirmaron que las mujeres tienen mayor probabilidad de sentir miedo al fracaso. Con respecto al miedo, se dice que este puede provocar cambios en el cerebro y otros órganos (Cacciotti y Hayton, 2015). Igualmente se tienen hallazgos de que el miedo al fracaso puede motivar la actividad empresarial, pero también está relacionado con el inventario de emociones negativas del individuo (Hunter, Jenkins y Mark-Herbert, 2020). Por último, los antecedentes familiares influyen de forma significativa en la intención emprendedora (Egerová, Eger y Mièík, 2017).

\section{Conclusiones}

Esta investigación ha logrado responder a las preguntas de investigación que indagaban sobre cuáles eran las percepciones que tenían los jóvenes sobre el fenómeno del emprendimiento en el Instituto Politécnico Nacional, dejando a la luz hallazgos que invitan a trabajar de forma incesante con acciones orientadas a desarrollar la mentalidad y el espíritu emprendedor de los estudiantes, lo que permitirá impulsar a los jóvenes en materia de emprendimiento. En este trabajo se encontró que siete de cada diez de los participantes se consideran emprendedores; este resultado incentiva a las escuelas a diseñar futuras estrategias que estimulen la creatividad y la innovación. Otro dato relevante que resulta esperanzador es que seis de cada diez estudiantes piensan que sus escuelas fomentan el emprendimiento, esto podría ser prometedor, sin embargo, lo que deja mucho que desear es que apenas uno de cada diez estudiantes ha participado en actividades relacionadas con el emprendimiento, lo que indica que tal vez los alumnos no muestran interés o están desmotivados con respecto al tema del desarrollo de la mentalidad emprendedora. 
Ahora bien, las implicaciones para las instituciones educativas sobre el fenómeno del emprendimiento son diversas, ya que en sus aulas se forman los futuros emprendedores; sin embargo, es un reto complejo. El emprendimiento necesita que los estudiantes posean una personalidad dotada de perseverancia, liderazgo y trabajo en equipo, por lo que resulta imprescindible trabajar en el desarrollo de esas habilidades. Por otra parte, las instituciones requieren programas de detección de talentos y dirigir las acciones afirmativas en esos grupos. Es importante que la comunidad estudiantil entienda que el emprendimiento requiere de esfuerzos extraordinarios, que necesita de la adquisición de conocimientos, tiempo y recursos.

Los vínculos con los sectores empresariales y las redes de emprendedores son de mucha utilidad, sin embargo, se necesita del apoyo de todos los actores involucrados para asesorar a los jóvenes que desean iniciarse en el emprendimiento; por medio del aprendizaje por experiencias los estudiantes pueden visualizar si ese mundo es para ellos y si tendrán la capacidad física y emocional para afrontar los retos a los que se enfrentarán. Por otra parte, los alumnos en el mundo empresarial pueden desarrollar ideas e identificar las nuevas tecnologías que se emplean.

Las escuelas tienen que voltear a mirar a las mujeres, que son las que sienten mayores miedos y dudas para emprender, por lo que la integración de programas con perspectiva de género podría coadyuvar a motivarlas y sensibilizarlas. Con respecto a los apoyos orientados al emprendimiento, los concursos son una adecuada opción que invita a los estudiantes con deseos de desarrollar sus ideas.

Los centros de emprendimiento son verdaderos semilleros de emprendedores que permiten orientar los esfuerzos, por lo que es fundamental ofrecer una difusión en la comunidad estudiantil y desarrollar acciones que mantengan los cursos y los apoyos que se les puedan brindar a los jóvenes, aún más en aquellos con escasos recursos económicos. Por último, en un futuro sería interesante realizar un comparativo con otras universidades públicas para poder identificar si son los mismos miedos que obstaculizan el emprendimiento o son diferentes, de esa forma se determinarían otras áreas susceptibles de mejora con respecto al emprendimiento universitario.

\section{REFERENCIAS}

Acs, Z. J., Szerb, L., y Autio, E. (2016) The global entrepreneurship and development index. En Global Entrepreneurship and Development Index 2015. SpringerBriefs in Economics. Cham: Springer. https://doi. org/10.1007/978-3-319-26730-2_2.

Alonso, P., Barrera, P., Barrón, S., Berrelleza, M., Carbajal, M., Cortés, J., Jiménez, V., Pouzou, R., y Rehák, J. (2019). El emprendimiento en el estado de Querétaro: análisis de los datos del Global Entrepreneuship Monitor 2019/2020. Instituto Tecnológico de Monterrey.
Anjum, T., Farrukh, M., Heidler, P., y Díaz Tautiva, J. A. (2021). Intención emprendedora: creatividad, emprendimiento y apoyo universitario. Revista de Innovación Abierta: Tecnología, Mercado y Complejidad, 7(1), 11.

Boubker, O., Arroud, M., y Ouajdouni, A. (2021). Entrepreneurship education versus management students' entrepreneurial intentions. A PLS-SEM approach. The International Journal of Management Education, 19(1), 100450. https://doi.org/10.1016/j. ijme.2020.100450. 
Brüne, N., y Lutz, E. (2020). The effect of entrepreneurship education in schools on entrepreneurial outcomes: A systematic review. Manag Rev Q, (70), 275-305. https:/ / doi.org/10.1007/s11301-019-00168-3.

Cacciotti, G., y Hayton, J. C. (2015). Fear and entrepreneurship: A review and research agenda. International Journal of Management Reviews, 17(2), 165-190.

Dionisio, E. A., Júnior, E. I., y Fischer, B. B. (2021). Country-level efficiency and the index of dynamic entrepreneurship: Contributions from an efficiency approach. Technological Forecasting and Social Change, 162, 120406. https://doi.org/10.1016/j.techfore.2020.120406.

Egerová, D., Eger, L., y Mièík, M. (2017). Does entrepreneurship education matter? Business students' perspectives. Tert Educ Manag, (23), 319-333. https://doi.org/1 $0.1080 / 13583883.2017 .1299205$.

Ekore, J. O., y Okekeocha, O. C. (2012). Fear of entrepreneurship among university graduates: a psychological analysis. International Journal of Management, 29(2), 515524.

Elert, N., y Henrekson, M. (2021). Entrepreneurship prompts institutional change in developing economies. Rev Austrian Econ, (34), 33-53. https://doi. org/10.1007/s11138-020-00501-0.

Fallah Haghighi, N., Mahmoudi, M., y Bijani, M. (2018). Barriers to entrepreneurship development in Iran's higher education: A qualitative case study. Interchange, (49), 353375. https://doi.org/10.1007/s10780-018-9330-9.

Fallah Haghighi, N., Razavi, S. M., Rezvanfar, A., y Kalantari, K. (2014). Evaluating faculty member's attitudes regarding to entrepreneurship obstacles in agricultural faculties of Iran. Journal of Organizational Culture Management, 12(2), 325-343.

Games, D. (2021, feb.). The effectiveness of mentoring and coaching in teaching business planning. En The 3rd International Conference on Educational Development and Quality Assurance (ICED-QA 2020) (pp. 1-5). Atlantis Press. https://doi.org/10.2991/assehr.k.210202.001.

GEM [Global Entrepreneurship Monitor] (2020). Diagnosis COVID 19, impacts on entrepreneurship, exploring policy remedies for recovery. GEM, London Business School.

Guojin, C. (2011). Study and practice on training scheme of university students' entrepreneurship ability. En Y. Wu (ed.), Computing and intelligent systems. ICCIC 2011. Communications in computer and information science, vol. 233. Berlín, Heidelberg: Springer. https:/ / doi.org/10.1007/978-3642-24010-2_41.
Hung, Q. (2020). Examining the influence of age and gender on entrepreneurship in Vietnam. The Journal of Asian Finance, Economics and Business (JAFEB), 7(10), 193-199. https://doi.org/10.13106/jafeb.2020.vol7. no10.193.

Hunter, E., Jenkins, A., y Mark-Herbert, C. (2020). When fear of failure leads to intentions to act entrepreneurially: Insights from threat appraisals and coping efficacy. International Small Business Journal, 39(5) 407423. https://doi.org/10.1177/0266242620967006.

Karimi, S., Chizari, M., Biemans, H., y Mulder, M. (2010). Entrepreneurship education in Iranian higher education: The current state and challenges. European Journal of Scientific Research, 48(1), 35-50.

Kirby, D. A. (2018). Entrepreneurship education: The need for a higher education revolution in the Arab World. En H. Fardoun, K. Downing y M. Mok (eds), The future of higher education in the Middle East and Africa. Cham: Springer. https:/ /doi.org/10.1007/978-3-31964656-5_8.

Kleine, K. (2020). Technology entrepreneurship, enriching entrepreneurship education. En M. Peters y R. Heraud (eds), Encyclopedia of educational innovation. Singapur: Springer. https://doi.org/10.1007/978981-13-2262-4_172-1.

Koyuncuoglu, D. (2021). An investigation of potential leadership and innovation skills of university students. International Journal of Education in Mathematics, Science and Technology, 9(1), 103-115. https://doi. org/10.46328/ijemst.1374.

Kozlinska, I. (2011). Current trends in entrepreneurship education: Challenges for Latvia and Lithuania. Social Research, 4(25), 75-88.

Liening, A., Geiger, J. M., Kriedel, R. y Wagner, W. (2016). Complexity and entrepreneurship: Modeling the process of entrepreneurship education with the Theory of Synergetics. En E. Berger y A. Kuckertz (eds.), Complexity in entrepreneurship, innovation and technology research. FGF Studies in Small Business and Entrepreneurship. Cham: Springer. https://doi.org/10.1007/9783-319-27108-8_5.

Lima, E., Almeida Lopes, R. M., Maria, V., Nassif, J., y da Silva, D. (2012). Opportunities to improve entrepreneurship education in higher education: Addressing Brazilian challenges. Proceedings of the Congress of the International Council of Small Business, New Zealand, June 2012.

Martínez-Martínez, S. L., y Ventura, R. (2020). Entrepreneurial profiles at the university: A competence 
approach. Frontiers in Psychology, 11, 3471. https:// doi.org/10.3389/fpsyg.2020.612796.

Mróz, A., y Ocetkiewicz, I. (2021). Creativity for sustainability: How do Polish teachers develop students' creativity competence? Analysis of research results. Sustainability, 13(2), 571.

Ndou, V., Secundo, G., y Mele, G. (2016). A processbased model for inspiring technology-driven entrepreneurship: An education perspective. En G. Passiante y A. Romano (eds), Creating technologydriven entrepreneurship. Londres: Palgrave Macmillan. https://doi.org/10.1057/978-1-137-59156-2_6.

Nicolaou, N., Phan, P. H., y Stephan, U. (2021). The biological perspective in entrepreneurship research. Entrepreneurship Theory and Practice, 45(1), 3-17. https://doi.org/10.1177/1042258720967314.

Nwekeaku, C. (2013). Entrepreneurship education and challenges to Nigerian universities. Journal of Education and Practice, 4(3), 51-56.

Pérez, M., Núñez, y Montoya, M. (2020). Perfil del emprendedor politécnico. En M. Pérez (coord.), Incubarpara innovar. 40 años de emprendimiento politécnico. Ediciones Díaz Santos.

Pérez, M. (2020). Incubar para innovar. 40 años de emprendimiento politécnico. Ediciones Díaz Santos.

Pisá-Bó, M., López-Muñoz, J. F., y Novejarque-Civera, J. (2021). The ever-changing socioeconomic conditions for entrepreneurship. International Entrepreneurship and Management Journal, 17, 1335-1355. https://doi. org/10.1007/s11365-020-00737-z.

Raposo, M., y do Paço, A. (2011). Special issue: entrepreneurship and education-links between education and entrepreneurial activity. International Entrepreneurship and Management Journal, 7, 143-144. https://doi. org/10.1007/s11365-010-0152-1.

Rivero, C. A. P., y Ubierna, F. (2021). The development of the entrepreneurial motivation from the university. International Entrepreneurship and Management Journal, 1-22. DOI: $10.1007 / \mathrm{s} 11365-020-00723-5$.
Sáfrányné Gubik, A., y Bartha, Z. (2021). Student perception and the efficacy of universities in shaping the entrepreneurial mindset. Theory Methodology Practice: Club Of Economics in Miskolc, 17(esp.), 65-76.

Seelig, T. (2015). Insight out: Get ideas out of your head and into the world. Nueva York: Harper Collins.

Shahin, M., Ilic, O., Gonsalvez, C., y Whittle, J. (2021). The impact of a STEM-based entrepreneurship program on the entrepreneurial intention of secondary school female students. International Entrepreneurship and Management Journal, 1-32. https://doi.org/10.1007/s11365-020-00713-7.

Vaquero-García, A., Ferreiro-Seoane, F. J., y Álvarez-García, J. (2017). Entrepreneurship and university: How to create entrepreneurs from university institutions. En M. PerisOrtiz, J. Gómez, J. Merigó-Lindahl y C. Rueda-Armengot (eds), Entrepreneurial universities. Innovation, technology, and knowledge management. Cham: Springer. https://doi. org/10.1007/978-3-319-47949-1_4.

Wyrwich, M., Stuetzer, M., y Sternberg, R. (2016). Entrepreneurial role models, fear of failure, and institutional approval of entrepreneurship: a tale of two regions. Small Bus Econ, 46, 467-492. https://doi.org/10.1007/s11187015-9695-4.

Xianming, W. (2013). Reflection on challenges and countermeasure of entrepreneurship education in China. CrossCultural Communication, 9(2), 18-22.

Yao, G., Xu, J., y Xu, J. (2013). An application of entrepreneurship score model in College student entrepreneurship education. En W. E. Wong y T. Ma (eds.), Emerging technologies for information systems, computing, and management. Lecture Notes in Electrical Engineering, vol 236. Nueva York: Springer. https://doi.org/10.1007/978-1-4614-70106_122.

Zeng, E. L. (2012). Research and practice of entrepreneurship education reform: The case of Wenzhou University. En L. Zhang y C. Zhang (eds.), Engineering education and management. Lecture Notes in Electrical Engineering, vol 111. Berlin, Heidelberg: Springer. https://doi. org/10.1007/978-3-642-24823-8_48.

Cómo citar este artículo:

Hernández Herrera, C. A., y Pérez Hernández, M. d. P. M. (2021). Los estudiantes politécnicos en México y sus percepciones sobre los miedos que los obstaculizan para emprender IE Revista de Investigación Educativa de la REDIECH, 12, e1295. doi: 10.33010/ie_rie_rediech.v12i0.1295. 